\title{
Safety and Efficacy of Hydroxychloroquine in COVID-19: A Systematic Review and Meta-Analysis
}

\author{
Waqas Ullaha, i, Hafez M. Abdullah ${ }^{\mathrm{b}}$, Sohaib Roomi ${ }^{\mathrm{a}}$, Yasar Sattarc, Talal Almas ${ }^{\mathrm{d}}$, \\ Smitha Narayana Gowda ${ }^{b}$, Rehan Saeed ${ }^{\mathrm{a}}$, Maryam Mukhtar ${ }^{\mathrm{e}}$, Ammar Ahmad $^{\mathrm{f}}$, \\ Tony Oliverb ${ }^{\mathrm{b}}$, M. Chadi Alraies ${ }^{\mathrm{g}}$, Donald C. Haas ${ }^{\mathrm{a}}$, David L. Fischman ${ }^{\mathrm{h}}$
}

\begin{abstract}
Background: During the initial phases of the coronavirus disease 2019 (COVID-19) epidemic, there was an unfounded fervor surrounding the use of hydroxychloroquine (HCQ); however, recently, the Centers for Disease Control and Prevention (CDC) has recommended against routine use of HCQ outside of study protocols citing possible adverse outcomes.
\end{abstract}

Methods: Multiple databases were searched to identify articles on COVID-19. An unadjusted odds ratio (OR) was used to calculate the safety and efficacy of HCQ on a random effect model.

Results: Twelve studies comprising 3,912 patients (HCQ 2,512 and control 1400) were included. The odds of all-cause mortality (OR: $2.23,95 \%$ confidence interval $(\mathrm{CI}): 1.58-3.13$, P value $<0.00001)$ were significantly higher in patients on HCQ compared to patients on control agent. The response to therapy assessed by negative repeat polymerase chain reaction (PCR) (OR: 1.83, 95\% CI: 0.50 $6.75, \mathrm{P}=0.36$ ), radiological resolution (OR: $1.98,95 \% \mathrm{CI}: 0.47$ - 8.36, P value $=0.36)$ and the need for invasive mechanical ventilation (IMV) (OR: 1.21, 95\% CI: $0.34-4.33$, P value $=0.76)$ were identical between the two groups. Overall, four times higher odds of net adverse events (NAEs) were observed in the HCQ group (OR: $4.59,95 \%$ CI $1.73-12.20$, $\mathrm{P}$ value $=0.02$ ). The measures for individual safety endpoints were also numerically lower in the control arm; however, none of these values reached the level of statistical significance.

Manuscript submitted May 22, 2020, accepted June 16, 2020

Published online July 4, 2020

aAbington Jefferson Health, Abington, PA 19001, USA

bUniversity of South Dakota, Vermillion, SD 57069, USA

'Icahn School of Medicine, Queens, NY, USA

dRoyal College of Surgeons, Dublin, Ireland

'Rawalpindi Institute of Cardiology, Rawalpindi, Pakistan

${ }^{f}$ Khyber Medical College, Peshawar, Pakistan

${ }^{g}$ Detroit Medical Center, Detroit, MI, USA

${ }^{\text {h}}$ Thomas Jefferson University, Philadelphia, PA, USA

${ }^{i}$ Corresponding Author: Waqas Ullah, Department of Internal Medicine, Abington Jefferson Health, 1200 Old York Rd, Abington, PA 19001, USA.

Email: waqasullah.dr@gmail.com

doi: https://doi.org/10.14740/jocmr4233
Conclusions: HCQ might offer no benefits in terms of decreasing the viral load and radiological improvement in patients with COVID-19. HCQ appears to be associated with higher odds of all-cause mortality and NAEs.

Keywords: COVID-19; SARS-CoV-2; HCQ; CQ

\section{Introduction}

On March 11, 2020, the World Health Organization (WHO) officially declared coronavirus disease 2019 (COVID-19) a global pandemic. Emerging as a handful of pneumonia case clusters in Wuhan, China, in late December 2019, COVID-19 has now reached all corners of the world. The epicenter of the outbreak has shifted over the past 4 months from China to Italy and now to New York. As of April 28, 2020, more than 3 million cases from over 180 countries and more than 200,000 deaths have been documented worldwide, with the USA accounting for nearly a quarter of all fatalities. The projected number of deaths in the USA is between 100,000 and 240,000, with an estimated total burden of over 2 million COVID-19 cases [1].

In response to this extraordinary public health challenge and the urgent need for medications to treat this disease, the Food and Drug Administration (FDA) established a Coronavirus Treatment Acceleration Program (CTAP) and issued Emergency Use Authorization (EUA) for chloroquine (CQ) and its derivative hydroxychloroquine (HCQ). CQ and HCQ (an analog of CQ) are primarily approved for the treatment of malaria and chronic inflammatory disorders (rheumatoid arthritis), respectively [2]. Scientific literature pertaining to the immunopathology of the severe acute respiratory syndrome (SARS) and Middle Eastern respiratory syndrome virus (MERS) has previously shown some effect of these medications against those viruses [3]. This lends some credence to the use of these medications against severe acute respiratory syndrome coronavirus 2 (SARS-CoV-2). Both drugs are thought to prevent the ingress of virions into the host cell, halt post-entry cascades and replication of the virus. The former is achieved by inhibition of the downstream glycosylation of angiotensin-converting enzyme 2 (ACE2) receptors, while en- 
dosomal acidification helps in reducing the overall virus burden [2-4].

Although HCQ was widely adopted for the treatment of COVID-19, this appears to have been based on little concrete evidence, but rather on anecdotal data emerging from China and France. While a few small-scale studies have demonstrated some favorable outcomes, the safety and efficacy of these regimens remain to be proven. This, along with considerable concern for HCQ-related adverse outcomes, has prompted us to conduct this meta-analysis as we await the results of randomized control trials (RCTs).

\section{Materials and Methods}

\section{Search strategy and data extraction}

The MEDLINE (PubMed, Ovid), EMBASE, Clinicaltrials. org and Cochrane databases were queried with various combinations of medical subject headings (MeSH) to identify relevant articles. There were no language or time restrictions placed. Backward snowballing was performed to retrieve unidentified studies that were missed on the initial search. The MeSH used included two subsets: one for the COVID-19 and another for HCQ. The two subsets of MeSH were combined in a 1:1 combination using Boolean operators. Results from all possible combinations were downloaded into an EndNote library. All RCTs and observational cohort studies (OCSs) until April 30, 2020, were evaluated. Studies comparing the safety and efficacy of the HCQ in COVID-19 were included. The efficacy endpoints were radiological resolution or decreased virological load on repeat polymerase chain reaction (PCR). Safety outcomes included net adverse events (NAEs), a composite of gastrointestinal, respiratory, neurological and dermatological outcomes. Secondary outcomes included components of NAE. Review articles, conference papers, and studies with no control arm or insufficient data were excluded.

\section{Data analysis}

The statistical analysis was performed using the DerSimonian and Laird test on a random-effects model to calculate an unadjusted odds ratio (OR). The probability value of $\mathrm{P}<0.05$ was considered statistically significant. The "test for overall effect" was reported as a $\mathrm{z}$-value corroborating the inference from the $95 \%$ confidence interval (CI). If the eligibility of a study was dubious or unduly influencing results due to its large sample size, a sensitivity analysis was performed. Subgroup analysis based on the type of control group (placebo vs. antiviral) was also performed. Higgins I-squared $\left(\mathrm{I}^{2}\right)$ statistical model was used to assess variations in outcomes of the included studies. $\mathrm{I}^{2}$ less than $40 \%$ corresponded to low heterogeneity. Depending upon the strength of evidence for heterogeneity ( $\mathrm{P}$ value from the Chi-square $\chi^{2}$ analysis), $\mathrm{I}^{2}$ of $41-74 \%$ indicated moderate (P $\geq 0.05)$ or moderate to severe $(\mathrm{P} \leq 0.05)$, and $\mathrm{I}^{2}$ of $75 \%$ or higher suggested substantial heterogeneity. Publication bias was il- lustrated graphically using a funnel plot. The methodological quality assessment of the included RCTs was performed using the Cochrane collaboration tool for the systematic review and meta-analysis, where each study was screened for five different types of bias (selection, performance, detection, attrition, and reporting bias). All statistical analysis was performed using the Digitize and the Cochrane Review Manager (RevMan) version 5.3 .

\section{Quality of the included studies}

The overall quality of the included RCTs was high (Fig. 1). Due to adequate randomization and allocation concealment, the risk of selection bias in RCTs was low. However, three retrospective cohort studies introduced a 30\% overall risk of selection bias. The risks of performance and detection bias were high because of inadequate blinding of participants and outcomes, respectively. Reporting bias across all studies was reduced due to an adequate description of the study results. The fact that most RCTs used an intention to treat model or had a minimal loss to follow-up of its participants, the risk of attrition bias was minimal.

An approval was obtained from the Institutional Review Board (IRB).

\section{Results}

\section{Search results and study characteristics}

The initial search revealed 351 articles. After removal of irrelevant and duplicate items, 160 articles were deemed relevant for full-text review. Of these, 148 articles were excluded based on our selection criteria. Fourteen articles (seven RCTs, seven OCSs) qualified for quantitative analysis [5-18]. The Preferred Reporting Items for Systematic Reviews and Meta-Analyses (PRISMA) flow diagram is shown in Figure 2.

A total of 3,912 patients, 2,512 in HCQ and 1,400 in the control arm were included. The mean age was 51 years, comprising 65\% male patients. Eight studies compared HCQ therapy to standard of care therapy (SCT) on outcomes including mortality, viral clearance and radiological resolution. SCT is defined as a supportive therapy with no specific treatment for COVID-19. The study of Borba et al compared high dose HCQ (600 mg twice daily) with low dose HCQ (450 mg twice daily) [9]. Huang et al compared HCQ against lopinavir/ritonavir therapy [8]. Carlucci et al compared HCQ + azithromycin + zinc with HCQ + azithromycin in the control group [14]. The baseline characteristics were comparable between the HCQ and the control group with a proportion of hypertension $(26 \%$ vs. $21.5 \%)$, diabetes mellitus ( $23 \%$ vs. $21 \%)$, smoking $(23.6 \%$ vs. $20.5 \%)$ and chronic kidney disease (11.7\% vs. $13 \%)$, respectively. The follow-up period varied from 6 days to 28 days with a median follow-up duration being 7 days. The detailed account of the inclusion criteria of studies and the baseline characteristics of included patients are given in Supplementary 


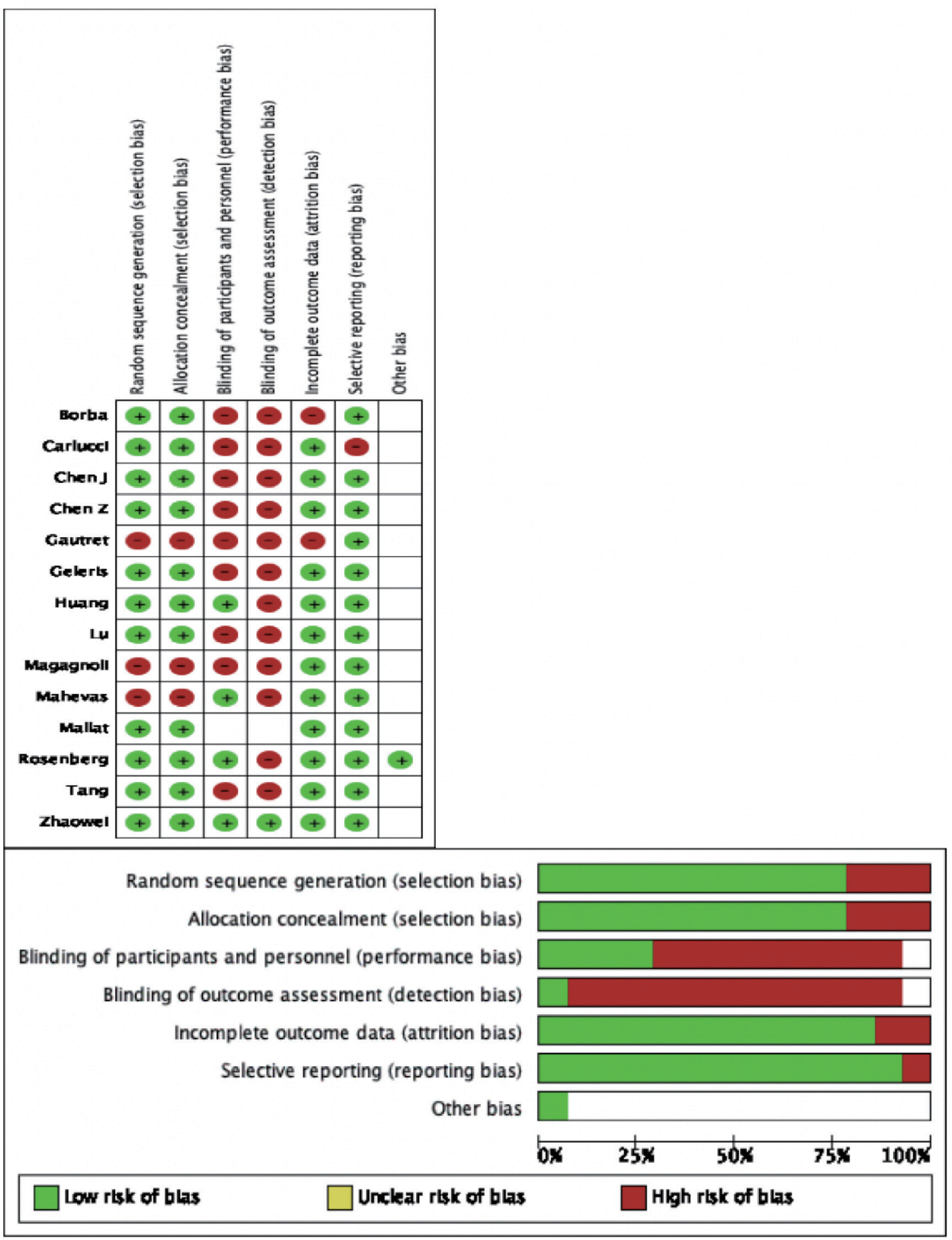

Figure 1. Methodological quality assessment of the included studies.

Material 1 (www.jocmr.org).

\section{Pooled analysis of efficacy endpoints}

A comprehensive pooled analysis of 12 studies did not show any benefit with the use of HCQ in any of the efficacy endpoints. Virological clearance assessed by negative repeat PCR (OR: $1.83,95 \% \mathrm{CI}: 0.50-6.75, \mathrm{P}=0.36$ ) and radiological resolution of concerning findings for COVID-19 (OR: 1.98, 95\% CI: $0.47-8.36, \mathrm{P}=0.36)$ were similar between the two groups. The in-hospital requirement for an invasive mechani- 


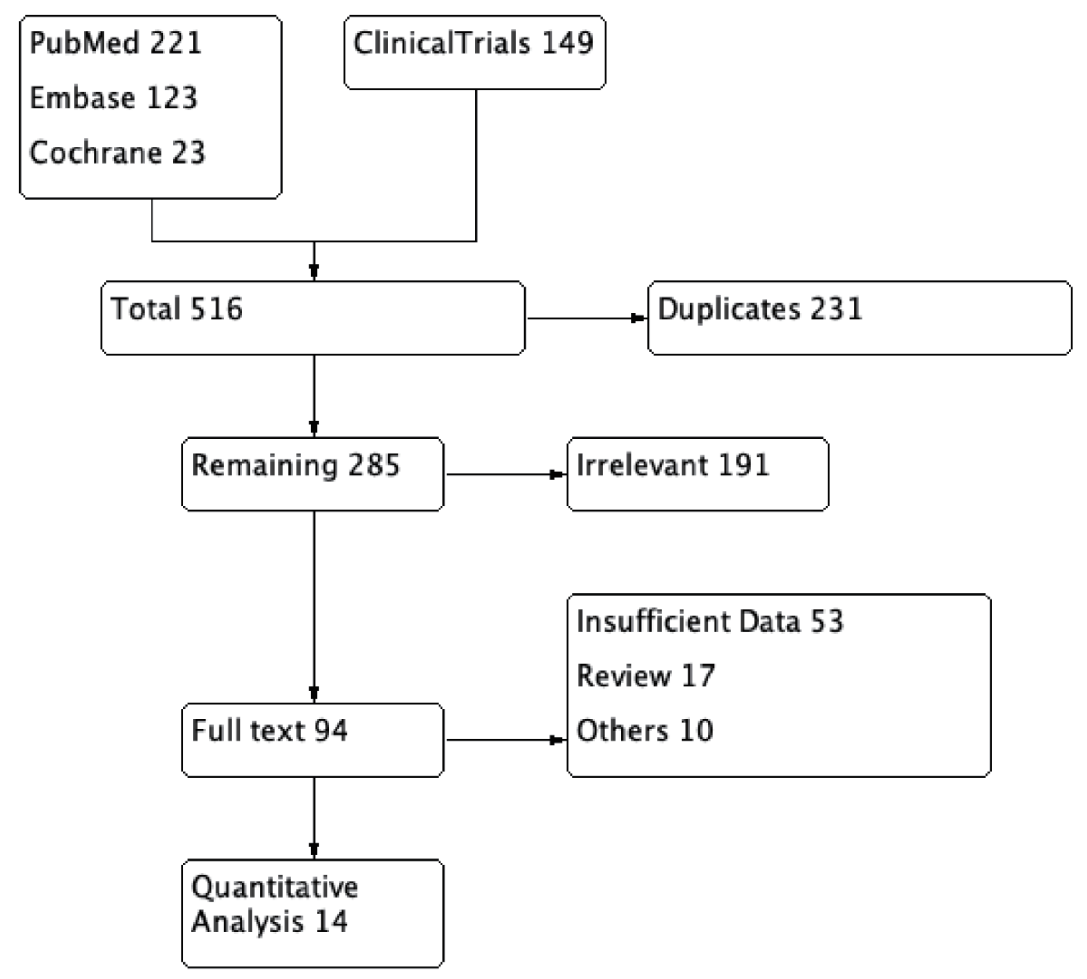

Figure 2. PRISMA flow diagram of the included studies in systematic review and meta-analysis. PRISMA: Preferred Reporting Items for Systematic Reviews and Meta-Analyses.

cal ventilation (IMV) (OR: $1.21,95 \% \mathrm{CI}: 0.34-4.33, \mathrm{P}=0.76)$ was also identical between patients on HCQ compared to those not on HCQ. There was no significant difference between the disease progression between the two groups (OR: $2.06,95 \%$ CI: $0.26-16.40, \mathrm{P}=0.50$ ), while, HCQ was found to be associated with significantly higher odds of all-cause mortality (OR: 2.23, 95\% CI: $1.58-3.13$, P value $<0.00001$ ) compared to patients in the control group. The heterogeneity in the outcomes of the included studies was moderate to high $\left(\mathrm{I}^{2}=54 \%\right.$ - 94\%) (Figs. 3, 4a).

\section{Pooled analysis of safety endpoints}

The incidence of NAE, a composite of all systemic complications, was used to gauge the safety of HCQ. The NAE with HCQ was significantly higher in the HCQ group as compared to control groups (OR: 4.59, 95\% CI: $1.73-12.20, \mathrm{P}$ value $=$ 0.02) (Fig. 4b). Interestingly, the odds of all individual adverse events were consistently higher but statistically nonsignificant in patients receiving HCQ for COVID-19; respiratory complications (OR: $1.19,95 \% \mathrm{CI}$ : $0.52-2.73$, P value $=0.68)$, gastrointestinal complications $(\mathrm{OR}: 1.45,95 \% \mathrm{CI}$ : $0.66-3.11, \mathrm{P}$ value $=0.35)$, neurological adverse symptoms (OR: 1.26, 95\% CI: $0.20-7.98$, P value $=0.81$ ), dermatological side effects (OR: 3.55, 95\% CI: 0.35 - 35.93, $\mathrm{P}=0.28$ ), cardiac arrest (OR: 0.88, 95\% CI: $0.33-2.39, \mathrm{P}$ value $=0.81)$ (Fig. 5). In the study of Borba et al [9], a high dose of HCQ was associated with a higher rate of QT prolongation, and ventricular arrhythmias.

\section{Subgroup sensitivity analysis}

Subgroup sensitivity analysis based on the type of medications used in combination with HCQ in the experimental group mirrored the overall findings of all efficacy endpoints except that mechanical ventilation was lower in the study by Carlucci et al [14], and control agent was favored in the study by Gautret et al [5] in terms of virological seroconversion. The safety profile was in concordance with the pooled results for all subgroups (Supplementary Materials 2 - 4, www.jocmr.org).

\section{Publication bias}

On visual assessment of the funnel plots, publication bias was minimal for overall outcomes (Fig. 6). The vertical axis of the plot used the standard error to estimate the sample size of the study; it is evident that the majority of the included studies fall at the bottom, indicating a small sample size. The horizontal spread reflected that most studies were underpowered due to a wide CI of the effect size.

\section{Discussion}

Our meta-analysis of 14 studies comprising 3,816 patients 
a

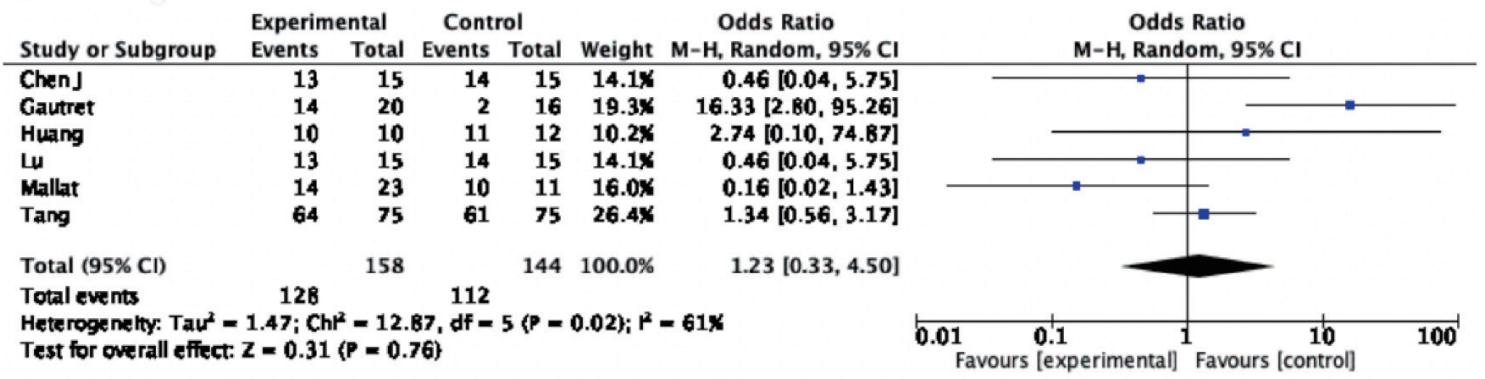

b

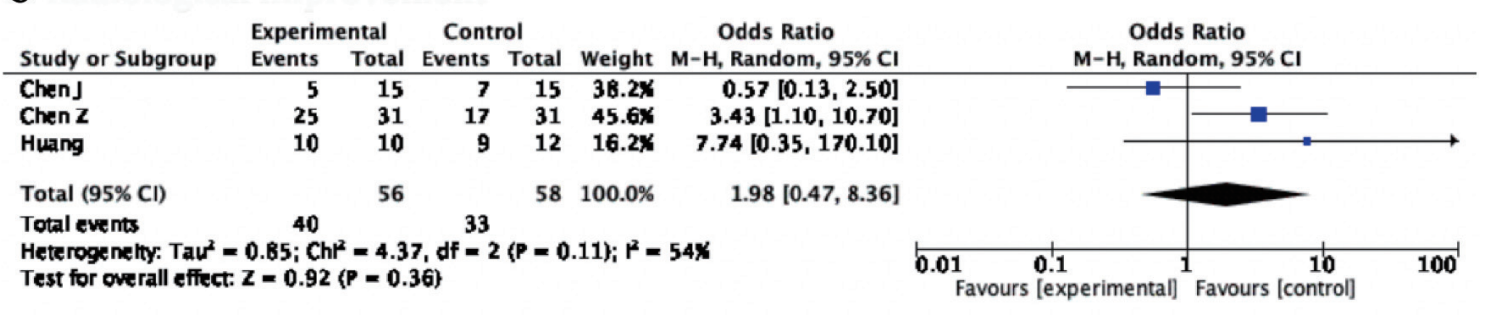

C

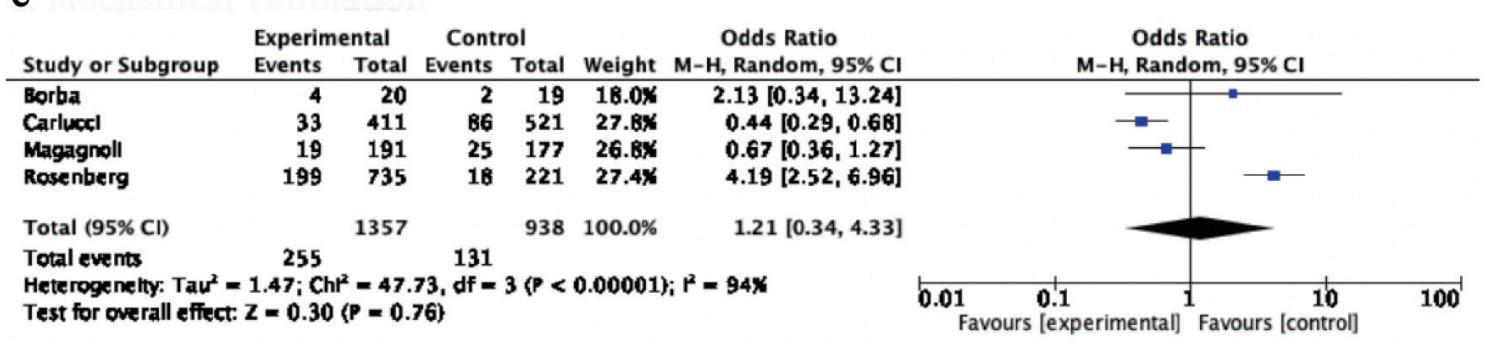

d

\begin{tabular}{|c|c|c|c|c|c|c|c|c|c|}
\hline \multirow{2}{*}{ Study or Subgroup } & \multicolumn{2}{|c|}{ Experimental } & \multicolumn{2}{|c|}{ Control } & \multirow{2}{*}{$\frac{\text { Weight }}{10.8 \times}$} & \multirow{2}{*}{$\begin{array}{l}\text { Odds Ratio } \\
\text { M-H, Random, } 95 \% \mathrm{Cl}\end{array}$} & \multicolumn{3}{|c|}{\begin{tabular}{c}
\multicolumn{1}{c}{ Odds Ratio } \\
$\mathrm{M}-\mathrm{H}$, Random, $95 \% \mathrm{CI}$
\end{tabular}} \\
\hline & 1 & 15 & 0 & 15 & & & & & \\
\hline Chen Z & 0 & 31 & 4 & 31 & $12.7 \times$ & $0.10[0.00,1.88]$ & & - & \\
\hline Gautret & 3 & 26 & 0 & 16 & $12.3 \times$ & $4.91[0.24,101.66]$ & & & \\
\hline Magagnoll & 19 & 191 & 25 & 177 & $51.5 \%$ & $0.67[0.36,1.27]$ & $\rightarrow$ & - & \\
\hline Zharowel & 0 & 31 & 4 & 31 & $12.7 \%$ & $0.10[0.00,1.8 B]$ & & & \\
\hline Total $(95 \% \mathrm{Cl})$ & & 294 & & 270 & $100.0 \%$ & $0.62[0.19,2.04]$ & & & \\
\hline Total events & 23 & & 33 & & & & & & \\
\hline $\begin{array}{l}\text { Heterogenelty: Taur } \\
\text { Test for overall effec }\end{array}$ & $\begin{array}{l}0.61 ; \mathrm{Ch} \\
\mathrm{z}=0.79\end{array}$ & $=5.8$ & $\mathrm{df}=4$ & & 21); $\mathrm{r}^{2}$ & & $0.01 \quad 0.1$ & ${ }_{\text {Favours [con }}^{1}$ & 100 \\
\hline
\end{tabular}

Figure 3. Forest plot for (a) virological cure, (b) radiological improvement, (c) ventilator required, and (d) progression to severe illness showing an individual and pooled OR for studies comparing HCQ-treated patients with control. OR: odds ratio; HCQ: hydroxychloroquine.

showed no significant difference in the efficacy of HCQ compared to the control arm, while the overall NAEs were five times higher in in the former group [5-16]. The individual safety measures including the incidence of gastrointestinal, respiratory, cardiac, dermatological and neurological complications were numerically higher but statistically non-significant between the two groups. Efficacy was gauged by a difference in the mortality rate, decrease in the viral load and radiological resolution of COVID-19-related findings. While there was no significant difference in most of the efficacy endpoints, patients on HCQ had a two-fold higher rate of in-hospital allcause mortality compared to patients on SCT.
Of the included studies, the major efficacy endpoint referred to the attainment of a negative PCR result, indicating total viral clearance usually at $5-10$ days of treatment. A pooled analysis of five studies comprising 268 patients revealed that a virologic cure was achieved in $84 \%$ of patients treated with HCQ compared to $77 \%$ in the control group. Gautret et al presented the results of a 14-day trial with 36 patients [5]. This was an outlier as the only study that had a statistically significant improvement in virologic cure rate. It is unclear if these findings could be attributed to the substantial amount of attrition and performance bias seen in this study [5]. Six patients on HCQ were prematurely excluded before completion of the 
a

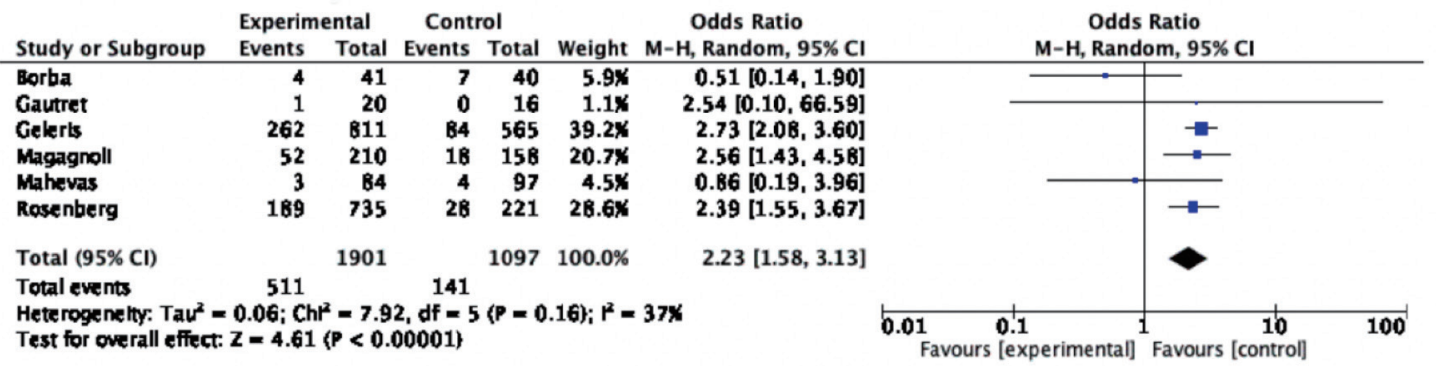

b

\begin{tabular}{|c|c|c|c|c|c|c|c|c|c|c|}
\hline Study or Subgroup & \multicolumn{2}{|c|}{ Experimental } & \multicolumn{2}{|c|}{ Control } & Weight & $\begin{array}{l}\text { Odds Ratio } \\
\mathrm{M}-\mathrm{H} \text {, Random, } 95 \% \mathrm{Cl}\end{array}$ & & \multicolumn{2}{|c|}{$\begin{array}{c}\text { Odds Ratio } \\
\mathrm{M}-\mathrm{H} \text {, Random, } 95 \% \mathrm{Cl}\end{array}$} & \\
\hline Chen Z & 2 & 31 & 0 & 31 & B.0\% & $5.34[0.25,115.89]$ & & & & \\
\hline Huang & 5 & 10 & B & 12 & $17.4 \%$ & $0.50[0.09,2.81]$ & & $\longrightarrow-$ & & \\
\hline $\begin{array}{l}\text { Tang } \\
\text { Zhaowel }\end{array}$ & $\begin{array}{r}15 \\
2\end{array}$ & $\begin{array}{l}70 \\
31\end{array}$ & $\begin{array}{l}3 \\
0\end{array}$ & $\begin{array}{l}80 \\
31\end{array}$ & $\begin{array}{r}22.8 \% \\
8.0 \%\end{array}$ & $\begin{array}{r}7.00[1.93,25.35] \\
5.34[0.25,115.89]\end{array}$ & & & & \\
\hline Total $(95 \% \mathrm{Cl})$ & & 892 & & 390 & $100.0 \%$ & $4.59[1.73,12.20]$ & & & & \\
\hline $\begin{array}{l}\text { Heterogeneity: Taur } \\
\text { Test for overall effec }\end{array}$ & $\begin{array}{l}66 ; C l \\
=3.05\end{array}$ & $=0$ & $\begin{array}{l}1, \mathrm{df}= \\
02)\end{array}$ & 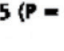 & & & 0.01 & 0.1 & 1 & 10 \\
\hline
\end{tabular}

Figure 4. Forest plot for (a) all-cause mortality, and (b) NAE, showing an individual and pooled OR for studies comparing HCQtreated patients with control. NAE: net adverse event; OR: odds ratio; $\mathrm{HCQ}$ : hydroxychloroquine.

study, and there were inconsistencies in the methods and centers of PCR testing between the two groups. Nevertheless, this study triggered massive interest and prompted other researchers to further evaluate the efficacy of HCQ. These subsequent studies have, however, shown an identical rate of virological clearance among both the HCQ-treated and SCT groups, mirroring the findings of our pooled analysis. It should be noted that the wide CI in our results indicates that it is difficult to draw definitive conclusions from the limited data currently available.

Three studies included in our analysis reported resolution of suggestive radiological findings on computerized tomography (CT) as an efficacy outcome [6-8]. A pooled analysis of these studies that comprised 56 (HCT) and 58 patients (SCT group) showed a trend favoring resolution in the HCQ group (71\% vs. 57\%), though statistical significance was not achieved. Huang et al was the only study that provided a breakdown of radiologic features. They reported a higher percentage of patients in the control group with the involvement of more than two lobes and also bilateral distribution of patchy shadows (92\% vs. $60 \%$ and $91 \%$ vs. $60 \%$, respectively) [8].

The most debilitating complication of severe acute respiratory syndrome coronavirus 2 (SARS-CoV-2) infection is acute respiratory failure necessitating the use of IMV and other concurrent resource-intensive needs in critical care units [10]. The need for artificial ventilation was reported by four studies, comprising 1,357 patients in the HCQ and 919 patients in the control group, showing $11 \%$ vs. $14 \%$ utilization, respectively, with a pooled OR of $0.86[9,10,14,15]$. While this favors the HCQ group, it was statistically not significant with $\mathrm{P}$ value of 0.24 . Among these four studies, we found that the study of Magagnoli et al was not only subject to the bias of nonrandomized data but also to ascertainment issues, i.e., sicker patients were more likely to receive HCQ on compassionate grounds and hence were more prone to have adverse outcomes [10].

Our mortality analysis of 3,004 (HCQ group: 1,907; control: 1,097) showed a significantly higher rate of all-cause mortality in patients in HCQ group compared to those on SCT. It should be noted, however, that this difference is principally driven by three out of 12 studies with higher population [1416]. Magagnoli et al reported a mortality rate three times higher in the HCQ group than the SCT group [10]. This finding is likely reflective of the inclusion of a sicker population in the treatment group, as evidenced by the higher rate of lymphopenia and azithromycin use. The study by Borba et al differed in terms of its inclusion criteria, comparing a high dose of HCQ against a low dose of HCQ [9]. Similarly, Carlucci et al studied the efficacy of HCQ, azithromycin, and zinc against HCQ and azithromycin, demonstrating increased frequency of discharges (OR: 1.53, 95\% CI: 1.12 - 2.09) and low mortality (OR: 0.449 , 95\% CI: $0.271-0.744$ ) in the zinc regimen arm [14]. On the contrary, Rosenberg et al compared SCT with HCQ and azithromycin, or HCQ alone, favoring the control arm due to a significantly lower need for IMV and mortality [15]. These findings were validated by Geleris et al [16]. Overall, the inconsistencies in the results of the included studies indicate that currently there is not enough data available to confirm or refute the efficacy of HCQ in this patient population, and we would have to wait for further studies to be reported before a more accurate assessment can be made [16].

While the therapeutic benefits of HCQ have now been widely touted, the adverse effects have not been adequately highlighted. In the current context, HCQ is generally thought to be safe due to a typically short course of therapy employed 
a

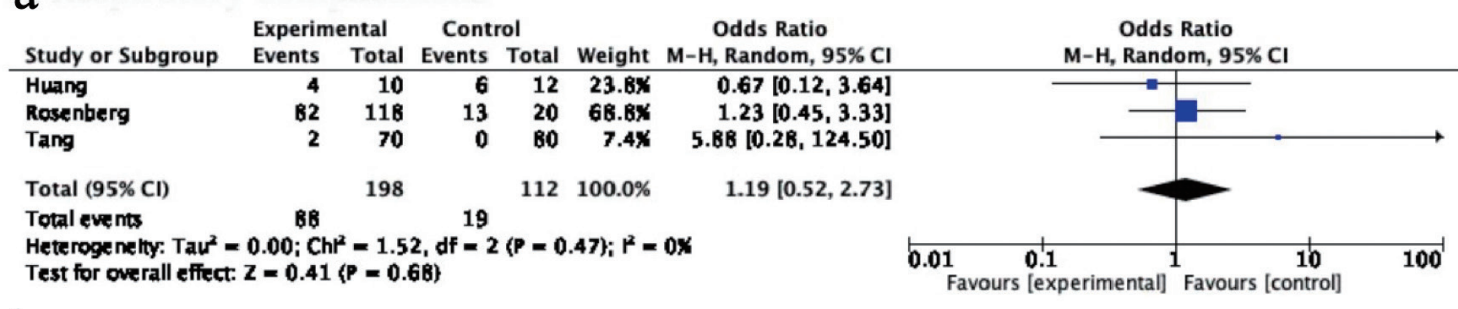

b

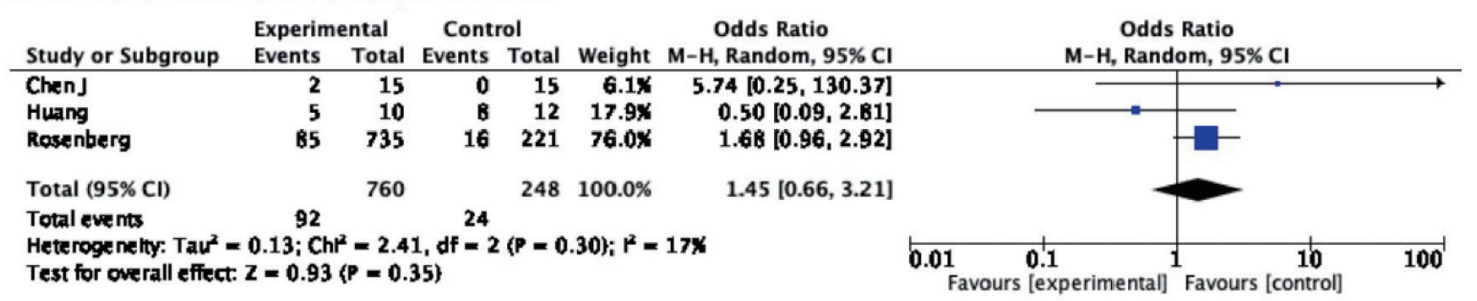

C

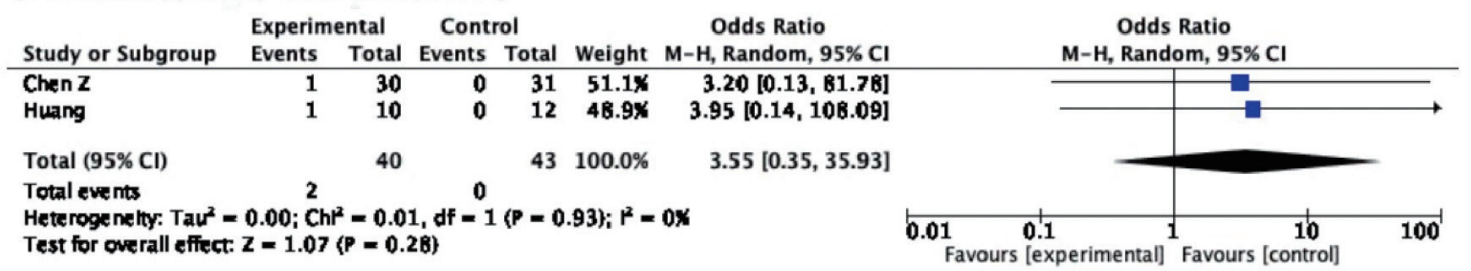

d

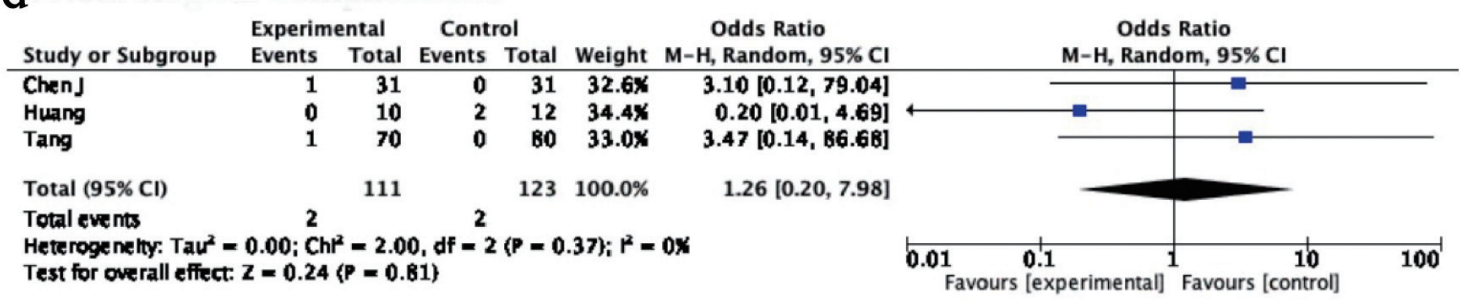

e

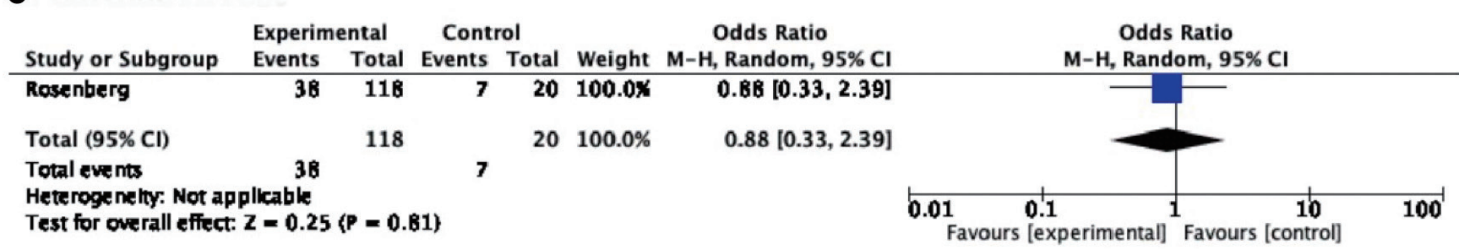

Figure 5. Forest plot for (a) respiratory complications, (b) gastrointestinal complications, (c) dermatological side effects, (d) neurological complications and (e) cardiac arrest showing an individual and pooled OR for all studies comparing HCQ treated patients with control. OR: odds ratio; HCQ: hydroxychloroquine.

for COVID-19. Our overall analysis showed a significantly higher incidence of NAEs in patients on HCQ. The individual components of the composite were also numerically higher in the HCQ arm. On analysis of 1,220 patients, only $29 \%$ of the SCT group developed adverse reactions compared to $77 \%$ of patients receiving HCQ. The neurological (1.8\% vs. 1.6\%), respiratory ( $7.5 \%$ vs. $6.5 \%)$, cardiac $(35 \%$ vs. $35 \%)$, and gastrointestinal $(18 \%$ vs. $8 \%)$ complications were higher in the HCQ vs. SCT, respectively. A study by Rosenberg et al reported a higher incidence of cardiac arrest with a combination of
HCQ and azithromycin (OR: 2.13, 95\% CI: 1.12 - 4.05) [15]. In light of these trends and with previous studies demonstrating major side effects of HCQ (cardiomyopathy, QT prolongation, and retinopathy), a considered risk to benefit determination should be made prior to HCQ use.

In summary, the available data regarding the effect of HCQ on safety and efficacy are limited and fraught with inconsistencies. A better analysis may be possible once more data become available from the numerous trials that are currently underway. However, it is important to keep in mind that the virologic cure 


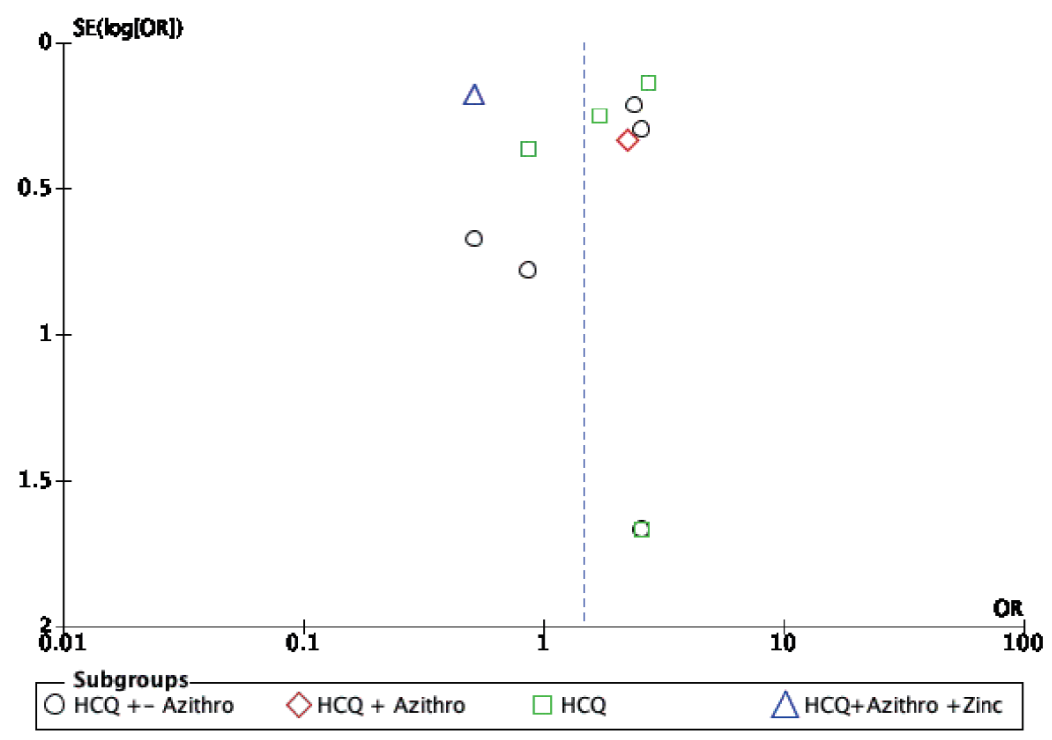

Figure 6. Funnel plot showing possible publication bias across all studies and subgroups.

does not necessarily correlate with the resolution of symptoms and patient outcomes. At present, our analysis shows that HCQ use is not associated with any benefit and could potentially be harmful.

\section{Limitations}

Our study is constrained by the limitations of the included studies. All studies were vastly underpowered due to the small sample size. There was significant heterogeneity in the inclusion criteria limiting our ability to perform subgroup analysis. Some therapeutic decisions were impacted by the prescriber's discretion, prioritizing treatment of the sicker population, potentially skewing results. Variability in the doses of HCQ, addition of antiviral agents to control arms, differing PCR testing methods and paucity of major RCTs call for caution while interpreting the results of this meta-analysis.

\section{Conclusions}

HCQ does not appear to offer significant benefits in terms of reducing the viral load, resolution of radiological findings, or progression of the COVID-19. HCQ might be associated with a higher mortality. Though there are trends in the data, pointing towards a higher complication rate with $\mathrm{HCQ}$, greater sample size and thus more statistically significant results, are needed to better inform the current debate. This meta-analysis supports the recommendation that HCQ should not be used outside of study protocols.

\section{Supplementary Material}

Suppl 4. Baseline Study Characteristics, Demographics and
Comorbidities Among Included Studies (Data Presented in Experimental/Control Group Pattern).

Suppl 2. Subgroups forest plot for ventilator required, virologic cure, and radiological improvement showing an individual and pooled OR for studies comparing HCQ-treated patients with control.

Suppl 3. Subgroup forest plot for all-cause mortality, sensitivity analysis of mortality, and progression to severe illness showing an individual and pooled OR for studies comparing HCQ-treated patients with control.

Suppl 4. Forest plot for net adverse events, respiratory complications, and cardiac arrest (left side); dermatologic complications, GI complications, and neurological complications (right side), showing an individual and pooled OR for all studies comparing HCQ-treated patients with control.

\section{Acknowledgments}

None to declare.

\section{Financial Disclosure}

None to declare.

\section{Conflict of Interest}

None to declare.

\section{Informed Consent}

Not applicable. 


\section{Author Contributions}

Conceptualization: Waqas Ullah. Data curation and formal analysis: Hafez M. Abdullah and Sohaib Roomi. Investigation and methodology: Ammar Ahmad. Project administration: M. Chadi Alraies and Tony Oliver. Resources, software, supervision and validation: David L. Fischman and Donald C. Haas. Writing of original draft: Yasar Sattar and Talal Almas. Writing review and editing: Maryam Mukhtar, Smitha Narayana Gowda, and Rehan Saeed.

\section{Data Availability}

The data supporting the findings of this study are available from the corresponding author upon reasonable request.

\section{References}

1. JHU. COVID-19 dashboard by the Center for Systems Science and Engineering (CSSE) at Johns Hopkins University (JHU). 2020.

2. Gao J, Tian Z, Yang X. Breakthrough: Chloroquine phosphate has shown apparent efficacy in treatment of COVID-19 associated pneumonia in clinical studies. Biosci Trends. 2020;14(1):72-73.

3. Downing NS, Aminawung JA, Shah ND, Braunstein JB, Krumholz HM, Ross JS. Regulatory review of novel therapeutics - comparison of three regulatory agencies. N Engl J Med. 2012;366(24):2284-2293.

4. Darrow JJ, Avorn J, Kesselheim AS. FDA Approval and Regulation of Pharmaceuticals, 1983-2018. JAMA. 2020;323(2):164-176.

5. Gautret P, Lagier JC, Parola P, Hoang VT, Meddeb L, Mailhe M, Doudier B, et al. Hydroxychloroquine and azithromycin as a treatment of COVID-19: results of an open-label non-randomized clinical trial. Int J Antimicrob Agents. 2020:105949.

6. Chen Z, Hu J, Zhang Z, Jiang S, Han S, Yan D, et al. Efficacy of hydroxychloroquine in patients with COVID-19: results of a randomized clinical trial. medRxiv. 2020;2020.03.22.20040758.

7. Chen J, Liu D, Liu L, Liu P, Xu Q, Xia L, Ling Y, et al. [A pilot study of hydroxychloroquine in treatment of patients with moderate COVID-19]. Zhejiang Da Xue Xue Bao Yi Xue Ban. 2020;49(2):215-219.

8. Huang M, Tang T, Pang P, Li M, Ma R, Lu J, Shu J, et al.
Treating COVID-19 with Chloroquine. J Mol Cell Biol. 2020;12(4):322-325.

9. Borba MGS, Val FdA, Sampaio VS, Alexandre MAA, et al. Chloroquine diphosphate in two different dosages as adjunctive therapy of hospitalized patients with severe respiratory syndrome in the context of coronavirus (SARS-CoV-2) infection: Preliminary safety results of a randomized, double-blinded, phase IIb clinical trial(CloroCovid-19 Study). medRxiv. 2020;2020.04.07.20056424.

10. Magagnoli J, Narendran S, Pereira F, Cummings T, Hardin JW, Sutton SS, Ambati J. Outcomes of hydroxychloroquine usage in United States veterans hospitalized with Covid-19. medRxiv. 2020.

11. Mahevas M, Tran V-T, Roumier M, Chabrol A, Paule R, Guillaud C, et al. No evidence of clinical efficacy of hydroxychloroquine in patients hospitalized for COVID-19 infection with oxygen requirement: results of a study using routinely collected data to emulate a target trial. medRxiv. 2020;2020.04.10.20060699.

12. Tang W, Cao Z, Han M, Wang Z, Chen J, Sun W, et al. Hydroxychloroquine in patients mainly with mild to moderate COVID-19: an open-label, randomized, controlled trial. medRxiv. 2020;2020.04.10.20060558.

13. Lu CC, Chen MY, Lee WS, Chang YL. Potential therapeutic agents against COVID-19: What we know so far. J Chin Med Assoc. 2020;83(6):534-536.

14. Carlucci P, Ahuja T, Petrilli CM, Rajagopalan H, Jones $\mathrm{S}$, Rahimian J. Hydroxychloroquine and azithromycin plus zinc vs hydroxychloroquine and azithromycin alone: outcomes in hospitalized COVID-19 patients. medRxiv. 2020;2020.05.02.20080036.

15. Rosenberg ES, Dufort EM, Udo T, Wilberschied LA, Kumar J, Tesoriero J, Weinberg P, et al. Association of Treatment With Hydroxychloroquine or Azithromycin With In-Hospital Mortality in Patients With COVID-19 in New York State. JAMA. 2020.

16. Geleris J, Sun Y, Platt J, Zucker J, Baldwin M, Hripcsak G, Labella A, et al. Observational Study of Hydroxychloroquine in Hospitalized Patients with Covid-19. N Engl J Med. 2020;382(25):2411-2418.

17. Mallat J, Hamed F, Balkis M, Mohamed MA, Mooty M, Malik A, et al. Hydroxychloroquine is associated with slower viral clearance in clinical COVID-19 patients with mild to moderate disease: A retrospective study. medRxiv. 2020:2020.04.27.20082180.

18. Zhaowei C, Jijia H, Zongwei Z, Shan J, Shoumeng H, Dandan Y, Ruhong Z, et al. Efficacy of hydroxychloroquine in patients with COVID-19: results of a randomized clinical trial. medRxiv. 2020:2020.03.22.20040758. 